\title{
ON THE SPOTTEDNESS AND MAGNETIC FIELD \\ OF T TAU-TYPE STARS
}

\author{
R. E. GERSHBERG \\ Crimean Astrophysical observctory
}

There are several observations that suggest the existence of surface heterogeneities on the T Tau-type stars. Firstly Dr. Ismailov frcm Shemakha has found strong variations of emission line profiles for 5 - 10 ninutes: 2-component profiles become 3 or 1 component and vice-versa. The results were confirmed recently in the sternberg Institute. Secondly, variations of polarization degree and relative intensities in the IR calcium triplet were found when the stellar brightness were being constant. Then, the relative intensities of the Ca triplet are not equal to what must be expected for both optically thin or thick but homogeneous emission regions. On the basis of the data Petrov and Sheberbakov from Crimean Observatory have proposed that $\mathrm{T}$ Tau stars have active regions with strong magnetic fields similar to stellar ones: they can give noticeable heterogeneities for calcium emission, for polarization effects in dust envelopes and local processes for profile variations. The existence of rather strong and local magnetic fields on $T$ Tauri stars may give a key for explaining the mysteries of these variables. Small variations of strong magnetic fields on stars with a convective zone can give a new mechanism for stellar variability. As it is known, only a quarter of the energy flux that reaches the bottom of the convective zone, is emitted from a sunspot in form of light and the main part of the energy escapes in form of hydromagnetic waves. Then, the sunspot exists if the field is not less than 1 kilogauss. Therefore the field acts as a relay: if the field is low we have light, if the field is strong we have mainly hydromagnetic vaves. The transition from low to high field may occupy only a smal.l part of the whole field range. The field variations on $T$ Tau stars can give strong brightness variations without additional energy sources but only by magnetic relay operation. I hope that this scheme gives a possibility to explain a set of features of $T$ Tau stars (such as their high ratio of chromospheric to photospheric radiation, IR excesses, the nature of their flares) as well as main features of FU Ori-type flares.

The full paper by Mr. Petrov and myself will be published in October in Astron.Zh. Letters.

Edith A. Müller (ed.), Highlights of Astronomy, Vol. 4, Part II, 407. All Rights Reserved. Copyright $(1977$ by the IAU. 